\title{
Erratum
}

\section{Synthesis of a xylo-Puromycin Analogue}

Benoît Y. Michel, Kollappillil S. Krishnakumar, Peter Strazewski* Synlett 2008, 2461.

In the advance online (e-First) publication of this manuscript, Scheme 1 was incorrect.

It has been corrected for both the print and the current online versions. 